\title{
Hipotermia en encefalopatía hipóxico-neonatal
}

\section{Hypothermia in neonatal hypoxic encephalopathy}

\author{
Ana Isabel Valdivielso-Martínez, María del Mar Alonso-Montejo, María del Carmen López-Castillo, Mercedes \\ Chaffanel-Peláez
}

\begin{abstract}
Resumen
ANTECEDENTES: La encefalopatía hipóxico-isquémica es una causa importante de daño neurológico agudo en el recién nacido. La hipotermia, inducida durante las primeras horas de vida, es un tratamiento eficaz para reducir la mortalidad y la discapacidad mayor en los niños que superviven a una agresión hipóxico-isquémica perinatal.

OBJETIVO: Describir las características de los pacientes con asfixia perinatal moderada a severa con protocolo de hipotermia terapéutica, la evolución del desarrollo psicomotor y las secuelas neurológicas.

PACIENTES Y MÉTODOS: Análisis descriptivo (noviembre 2011 -marzo 2017), retrospectivo, de las hospitalizaciones urgentes de recién nacidos que recibieron hipotermia activa corporal total. Se recabaron los datos clínicos, tratamiento, pruebas complementarias, complicaciones y secuelas neurológicas.

RESULTADOS: Se reunieron 51 casos, de los que 30 sufrieron encefalopatía severa y 21 moderada, según la escala de García Alix. Los hallazgos de resonancia magnética craneal fueron compatibles con encefalopatía hipóxico-isquémica leve $(n=9)$, moderada $(n=4)$ y severa $(n=19)$. En 14 pacientes no se registraron hallazgos de encefalopatía hipóxico-isquémica. Sucedieron 16 fallecimientos, 15 enseguida de la adecuación del esfuerzo terapéutico. En la actualidad permanecen en consulta externa 15 pacientes con secuelas neurológicas; 8 se dieron de alta por estar asintomáticos y 6 se perdieron en el seguimiento.

CONCLUSIONES: La terapia con hipotermia controlada parece segura y ha demostrado mejor evolución de los pacientes con encefalopatía hipóxico-isquémica moderadasevera.

PALABRAS CLAVE: Encefalopatía hipóxico-isquémica; hipotermia del recién nacido; tratamiento eficaz; niños; asfixia perinatal; hipotermia terapéutica; Neurología pediátrica.
\end{abstract}

Abstract

BACKGROUND: Hypoxic-ischemic encephalopathy is a major cause of acute neurological damage in the newborn. Hypothermia induced during the first hours of life of the newborn is an effective treatment to reduce mortality and major disability in children who survive after perinatal hypoxic-ischemic insult.

OBJECTIVE: Describe the characteristics of patients with moderate to severe perinatal asphyxia with therapeutic hypothermia protocol; as well as evolution of psychomotor development and neurological sequelae.

MATERIAL AND METHODS: Retrospective descriptive analysis of urgent hospitalizations of newborns who received therapy with active total body hypothermia. Clinical data, precise treatment, complementary tests, complications and neurological sequelae were collected.

RESULTS: 51 cases were collected, of which 30 suffered from severe encephalopathy and 21 from moderate, according to the García Alix scale. Cranial MRI findings were consistent with mild $(n=9)$, moderate $(n=4)$, and severe $(n=19)$ hypoxic-ischemic encephalopathy. In 14 patients, no findings of hypoxic-ischemic encephalopathy were recorded. There were 16 deaths, 15 immediately after the adequacy of the therapeutic effort. At present, 15 patients with neurological sequelae remain in outpatient care; 8 were discharged because they were asymptomatic. 6 patients were lost to follow-up. CONCLUSIONS: Therapy with controlled hypothermia seems safe, demonstrating an improvement in the evolution of patients with moderate-severe hypoxic-ischemic encephalopathy.

KEYWORDS: Hypoxic ischemic encephalopathy; Newborn Hypothermia; Effective treatment; Children; Perinatal asphyxia; Therapeutic hypothermia; Pediatric Neurology.
Hospital Regional Universitario Carlos Haya, Málaga, España.

Recibido: 23 de abril 2020

Aceptado: 21 de octubre 2020

Correspondencia

María del Mar Alonso Montejo

marialonsom9@gmail.com

Este artículo debe citarse como Valdivielso-Martínez Al, Alonso-Montejo MM, López-Castillo MC, ChaffanelPeláez M. Hipotermia en encefalopatía hipóxico-neonatal. Acta Pediatr Méx 2020; 241-8. 


\section{ANTECEDENTES}

La encefalopatía hipóxico-isquémica es una causa importante de daño neurológico agudo en el recién nacido a término, o casi a término. Su incidencia en los países desarrollados es baja; en España afecta, aproximadamente, a 1 a 3 casos por cada 1000 recién nacidos vivos de más de 36 semanas de gestación. ${ }^{1}$ En la actualidad, la encefalopatía hipóxico-isquémica es causa de $23 \%$ de las muertes neonatales. ${ }^{2}$

En el trascurso del siglo XX no se dispuso de tratamiento específico alguno para prevenir o aminorar el daño cerebral asociado con la agresión cerebral hipóxico-isquémica. Por fortuna, en la última década, diversos ensayos clínicos Ilevados a cabo en Europa, Australia y Estados Unidos han demostrado que la reducción de la temperatura corporal en $3-4{ }^{\circ} \mathrm{C}$, mediante enfriamiento corporal total o selectivo de la cabeza, aplicado tempranamente y mantenido durante 72 h es una intervención eficaz para reducir la mortalidad y la discapacidad mayor en los niños que superviven a una agresión hipóxicoisquémica perinatal. ${ }^{3,4,5}$

El objetivo de este estudio fue: describir las características de los pacientes con asfixia perinatal moderada a severa con protocolo de hipotermia terapéutica; la evolución del desarrollo psicomotor y las secuelas neurológicas.

\section{PACIENTES Y MÉTODOS}

Análisis descriptivo (noviembre 2011-marzo 2017) y retrospectivo de las hospitalizaciones urgentes de recién nacidos que recibieron terapia con hipotermia activa corporal total. Se recopilaron datos de: la historia clínica, antecedentes perinatales, terapia para la encefalopatía hipóxico-isquémica moderada o severa que siguió los criterios clínicos y diagnósticos de la escala de García-Alix ${ }^{6}$ y la modificada de Sarnat. ${ }^{7}$ Todos los datos se trataron en forma anónima después de obtener el consentimiento informado del padre o la madre.

Variables recopiladas: evento centinela, registro tococardiográfico, edad gestacional, peso al nacimiento, puntuación Apgar al minuto y 5 minutos, tipo de reanimación practicada, grado de encefalopatía hipóxico-isquémica (moderada o grave), convulsiones antes o durante la terapia con hipotermia o el recalentamiento; tratamiento con fármacos antiepilépticos, sedación, soporte respiratorio y cardiocirculatorio, complicaciones clínicas o analíticas, grado de afectación radiológica en la resonancia magnética practicada a las dos semanas de vida siguiendo la clasificación de Martínez-Biarge y colaboradores, ${ }^{8}$ patrón registrado en el electroencefalograma integrado por amplitud, evolución del desarrollo psicomotor y de secuelas neurológicas.

El primer año, el seguimiento del desarrollo psicomotor se hizo cada 3 meses y, posteriormente, cada 6 meses hasta los 3 años. También se consideraron los dados del alta de Neuropediatría a los 3 años si permanecían asintomáticos. En caso de secuelas neurológicas la periodicidad de las revisiones se determinó individualmente.

\section{RESULTADOS}

Se estudiaron 51 casos con edad gestacional media de $38 \pm 6$ semanas y media de peso de 3041 g. La puntuación media en la prueba de Apgar al minuto fue de 2.6 y 4.5 a los 5 minutos. Se aplicaron maniobras de reanimación con masaje cardiaco y fármacos a 26 de los 51 neonatos; se intubaron 17 de 51 y recibieron ventilación con presión positiva intermitente 6 de 51 . En cuanto al evento centinela, en 33 casos se trató de un 
estado fetal no tranquilizador (incluidos 9 desprendimientos de placenta, 1 prolapso de cordón y 1 caso de rotura uterina materna), seguido de distocia de parto en 6 pacientes, colapso neonatal precoz en 4 (agresión hipóxico-isquémica postnatal), 2 circulares de cordón y un neonato nació en situación de paro cardiorrespiratorio. Sufrieron encefalopatía severa 30 de los 51, en 21 moderada según la escala de García Alix. ${ }^{6}$ En el Cuadro 1 se describen las complicaciones clínico-analíticas durante la terapia con hipotermia.

Antes del inicio de la hipotermia convulsionaron 20 pacientes: 18 durante y 2 en el recalentamiento. En 13 pacientes las convulsiones se controlaron con fenobarbital intravenoso, fue necesario añadir midazolam en 3 pacientes, fenitoína en 2 y un caso se controló con este único fármaco.

Todos los neonatos requirieron sedación para minimizar el estrés térmico; posteriormente tuvieron bradicardia sinusal de 70-100 lpm.

Cuadro 1. Complicaciones clínico-analíticas durante la terapia con hipotermia

\begin{tabular}{ll}
\hline Crisis convulsivas & $18(35 \%)$ \\
\hline Hipertensión pulmonar & $19(37 \%)$ \\
\hline Disfunción ventricular cardiaca & $4(8 \%)$ \\
\hline Insuficiencia respiratoria con VMI & $48(94 \%)$ \\
\hline Alteraciones en la glucemia & $39(76 \%)$ \\
Hipoglucemia & $16(31 \%)$ \\
& Hiperglucemia \\
& $21(41 \%)$ \\
\hline Insuficiencia renal aguda con TRR & $8(16 \%)$ \\
Diálisis peritoneal & $7(13 \%)$ \\
Hemofiltración veno-venosa & $1(2 \%)$ \\
Coagulopatía $\left(\mathrm{TP}^{3}<60 \%\right)$ & $48(94 \%)$ \\
\hline $\begin{array}{l}\text { Trombopenia } \\
\left(<100.000 \text { plaq } / \mathrm{mm}^{3}\right)\end{array}$ & $21(41 \%)$ \\
\hline
\end{tabular}

'Ventilación mecánica invasiva

${ }^{2}$ Terapia reemplazo renal

${ }^{3}$ Tiempo de protrombina
En todos los casos se practicó ecocardiografía y se detectó disfunción ventricular en 4 e hipertensión pulmonar en 19. Se reportó que 38 pacientes requirieron apoyo con fármacos vasoactivos.

A 48 pacientes se les dio soporte respiratorio con ventilación mecánica invasiva, de los que 7 requirieron ventilación de alta frecuencia; en 6 pacientes pudo procederse a la extubación selectiva durante el procedimiento de hipotermia.

En 39 pacientes se registraron alteraciones de la glucemia, en uno de ellos la hiperglucemia no remitió a pesar del descenso de los aportes de glucosa; 10 pacientes requirieron aporte de insulina y 12 se encontraron en estado hipoglucémico.

Ocho pacientes requirieron terapia de reemplazo renal (7 diálisis peritoneal 1 hemofiltro). En 48 pacientes se registró coagulopatía con un tiempo de protrombina inferior al $60 \%$ y 21 con trombopenia por debajo de 100 x 109/L. En ningún caso se registró quemadura térmica.

En el Cuadro 2 se resume la evaluación del grado de afectación del daño neurológico mediante pruebas complementarias.

En 10 pacientes el patrón del electrocardiograma fue continuo, con voltaje normal, en 16 patrón discontinuo con voltaje normal, en 7 bajo voltaje, en 6 brote-supresión y 5 patrón plano.

Los hallazgos de la resonancia magnética craneal fueron compatibles con encefalopatía hipóxicoisquémica leve en 9 neonatos, moderada en 4 y severa en 19. En 14 pacientes no hubo hallazgos de encefalopatía hipóxico-isquémica. A los 5 pacientes restantes no se les practicó la resonancia magnética craneal porque fallecieron antes de la prueba. Cuadro 3 
Fallecieron 16 pacientes, 15 después de la adecuación del esfuerzo terapéutico. En la actualidad permanecen en consulta externa 15 pacientes con secuelas neurológicas (Cuadro 3); 8 se dieron de alta por estar asintomáticos y 6 se perdieron en el seguimiento.

De los 4 pacientes con encefalopatía hipóxicoisquémica por colapso neonatal temprano, 2 fallecieron y 2 tuvieron un desarrollo psicomotor adecuado a los 3 años. En 4 pacientes se llegó a un diagnóstico final, adicional al de encefalopatía hipóxico-isquémica, de contusión medular secundaria a parto traumático, déficit parcial
Cuadro 2. Resultados de las pruebas complementarias de evaluación del estado neurológico

\section{Patrón de EEG ${ }^{1}$ integrado por amplitud}

Continuo voltaje normal

$10(20 \%)$

Discontinuo voltaje normal

$16(31 \%)$

Bajo voltaje

$7(14 \%)$

Brote-supresión

$6(12 \%)$

Plano

$5(10 \%)$

Grado de afectación en la resonancia magnética

Encefalopatía hipóxico-isquémica leve $\quad 9$ (18\%)

Encefalopatía hipóxico-isquémica moderada 4 (8\%)

Encefalopatía hipóxico-isquémica severa

$19(37 \%)$

Electroencefalograma.

Cuadro 3. Antecedentes y evolución neurológica de los pacientes con secuelas neurológicas tratados con hipotermia corporal total

\begin{tabular}{|c|c|c|c|c|}
\hline Edad actual & $\begin{array}{l}\text { Encefalopatía } \\
\text { neonatal }\end{array}$ & Patrón en $E E G^{1}$ & $\begin{array}{l}\text { Hallazgos de la resonancia } \\
\text { magnética a las } 2 \text { semanas }\end{array}$ & Evolución \\
\hline 18 meses & Moderada & Discontinuo & $\begin{array}{l}\text { Pequeño sangrado } \\
\text { parenquimatoso }\end{array}$ & Retraso psicomotor \\
\hline 18 meses & Grave & Plano-hipoactivo & $\begin{array}{l}\text { Esquizencefalia de labio } \\
\text { cerrado. }\end{array}$ & $\begin{array}{c}\text { Tetraparesia espástica. } \\
\text { Encefalopatía multiquística. }\end{array}$ \\
\hline 19 meses & Moderada & Discontinua & $\begin{array}{l}\text { Hemorragia intra y periventricular. } \\
\text { Necrosis cortical laminar. }\end{array}$ & Hipotonía global \\
\hline 20 meses & Moderada & Brote-supresión & Hemorragia intraventricular & $\begin{array}{l}\text { Hipotonía global. Déficit } \\
\text { de piroxina. }\end{array}$ \\
\hline 20 meses & Grave & Bajo voltaje & Afectación de $\mathrm{GB}^{2}$ y tálamo & Tetraparesia espástica \\
\hline 21 meses & Moderada & Discontinuo & $\begin{array}{l}\text { Necrosis cortical laminar. } \\
\text { Hematoma subgaleal. }\end{array}$ & $\begin{array}{l}\text { Hipotonía axial, hipertonía } \\
\text { de EEII }\end{array}$ \\
\hline 22 meses & Grave & Bajo voltaje & Normal & Leve hipertonía de EEII \\
\hline 24 meses & Grave & Discontinuo & Leve isquemia GB & $\begin{array}{c}\text { Parálisis cerebral discinética. } \\
\text { Gastrostomía. }\end{array}$ \\
\hline 24 meses & Grave & Discontinúo & Pequeña alteración $\mathrm{SB}^{1}$ cortical & Retraso del lenguaje \\
\hline 36 meses & Grave & Discontinuo & $\begin{array}{c}\text { Alteración } \mathrm{SB}^{1} \text { bilateral. } \\
\text { Hemorragia } \\
\text { puntiforme en la cápsula interna. }\end{array}$ & Hipoacusia izquierda \\
\hline 36 meses & Grave & Bajo voltaje & $\begin{array}{l}\text { Lesiones isquémicas en } \mathrm{SB}^{1} \text {, daño } \\
\text { hemorrágico grave y necrosis laminar. }\end{array}$ & $\begin{array}{l}\text { Retraso en la maduración. } \\
\text { Hipoacusia derecha. }\end{array}$ \\
\hline 48 meses & Moderada & Continuo & Afectación GB² & Precisa apoyo para caminar \\
\hline 48 meses & Grave & Bajo voltaje & Alteración $\mathrm{GB}^{2}$ y en el tálamo & Tetraparesia espástica \\
\hline 60 meses & Moderada & Discontinuo & Leve afectación en la cápsula interna & $\begin{array}{l}\text { Hemiparesia derecha. } \\
\text { Crisis } \\
\text { parciales. }\end{array}$ \\
\hline 60 meses & Grave & Bajo voltaje & Alteración difusa $\mathrm{SB}^{1}$ bilateral & $\begin{array}{l}\text { Tetraparesia flácida. } \\
\text { Hipoacusia. } \\
\text { Déficit visual. }\end{array}$ \\
\hline
\end{tabular}

SB1: sustancia blanca; GB2: ganglios de la base; EEG: electroencefalograma; RM: resonancia magnética; Ell: extremidades inferiores. 
del factor XI de la coagulación con hemorragias subaracnoidea y corticales secundarias, déficit de piridoxina y esquizencefalia de labio cerrado, respectivamente.

\section{DISCUSIÓN}

Pese a los grandes progresos en la Medicina y Perinatología, la encefalopatía hipóxico-isquémica neonatal sigue siendo un problema en nuestro medio. Hasta hace unos años, la única terapia disponible para los neonatos con encefalopatía hipóxico-isquémica era la de soporte. Por fortuna, en las últimas décadas diversos ensayos clínicos, Ilevados a cabo en Europa, Australia y Estados Unidos han demostrado que la reducción de la temperatura corporal en $3-4{ }^{\circ} \mathrm{C}$ mediante enfriamiento corporal total o selectivo de la cabeza, iniciada tempranamente (antes del término de las 6-8 horas de vida) y mantenida durante 72 horas es una intervención eficaz para reducir la mortalidad y la discapacidad mayor en los niños que superviven a un episodio hipóxico-isquémico perinatal. ${ }^{3,4,5}$

En los últimos años se están llevando a cabo diversos ensayos clínicos en la búsqueda de un tratamiento farmacológico coadyuvante que, junto con la hipotermia, consiga aminorar el daño cerebral producido luego de la hipoxia; destacan por sus resultados prometedores: ácido valproico, ${ }^{9}$ magnesio, ${ }^{10}$ alopurinol ${ }^{11}$ y topiramato. ${ }^{12,13}$

Estudios recientes in vitro y en animales proponen la modulación terapéutica de la neurogénesis con eritropoyetina, ${ }^{14}$ células madre mesenquimales o cannabinoides. ${ }^{15}$ No obstante, se requieren más estudios clínicos, antes de incorporarlos como terapia aislada o coadyuvante en neonatos con hipotermia por encefalopatía hipóxico-isquémica.
Otra línea de investigación que está en pleno auge son los marcadores biológicos de daño neurológico en neonatos con encefalopatía hipóxico-isquémica: citocinas proinflamatorias, proteína SB100, troponinas, proteína dickkopf 1 y las concentraciones de selenio. ${ }^{16}$ También, recientemente, se han relacionado el descenso de gelsolina plasmática y de amiloide $\beta 42$ y 44 con el grado de encefalopatía hipóxico-isquémica. Está pendiente de determinar su valor pronóstico a mediano y largo plazo. ${ }^{17}$

En nuestra serie 39 de 51 pacientes sufrieron alteraciones de la glucemia, lo que se ha relacionado con peor pronóstico neurológico a los 18 meses. ${ }^{18}$

Los episodios sumamente letales o el "colapso neonatal temprano" suponen una lesión anóxica al cerebro de los recién nacidos a término, y uno de los máximos factores de riesgo para que se produzcan es la práctica de piel con piel, sin supervisión o acompañamiento de la madre, ${ }^{19}$ como ocurrió en cuatro pacientes de la serie. La similitud en el mecanismo de producción del daño cerebral (hipoxia, isquemia) y sus secuelas clínicas y hallazgos en resonancia magnética posteriore ${ }^{20}$ han motivado el reporte de numerosos casos clínicos ${ }^{21-24}$ en los que se inicia la terapia neuroprotectora con hipotermia, como se hizo en los pacientes de esta serie. Los resultados son inciertos y no está establecida la indicación de la terapia con hipotermia en estos supuestos, porque faltan ensayos clínicos al respecto. En la serie aquí reportada 2 de 4 pacientes fallecieron y 2 de 4 no tuvieron secuelas neurológicas a los 3 años de vida.

Existen otras patologías neurológicas con manifestación en la etapa neonatal temprana que podrían beneficiarse con la hipotermia, lo mismo que el colapso neonatal temprano, el infarto 
cerebral o la encefalopatía hipóxico-isquémica en prematuros tardíos. ${ }^{25}$ En la serie de casos aquí reportada hubo 4 pacientes que iniciaron con síntomas neurológicos agudos moderados o severos después de un evento "centinela" periparto o un registro tococardiográfico no tranquilizador; por eso se inició la terapia con hipotermia corporal total. Sin embargo, los exámenes de laboratorio o de imagen revelaron otros diagnósticos que pudieran justificar los síntomas neurológicos del neonato: contusión medular secundaria a parto traumático, déficit parcial del factor XI de la coagulación con hemorragia subaracnoidea y cortical secundarias, déficit de piridoxina y esquizencefalia del labio cerrado.

La serie aquí reportada es consistente con lo publicado en relación con el patrón del electrocardiograma integrado por amplitud, que es sumamente predictivo del mal desenlace clínico relacionado con el desarrollo psicomotor ${ }^{26}$ y con la afectación "severa" en la resonancia magnética; es decir, la afectación de los ganglios de la base, sobre todo el brazo posterior de la cápsula interna, se asocia estrechamente con las secuelas motoras; su máxima expresión es la tetraparesia espástica. ${ }^{27-30}$ Hace poco se publicó una nueva escala capaz de predecir daño neurológico en neonatos que han sufrido asfixia perinatal tratados con hipotermia, según la afectación en la resonancia magnética aguda. ${ }^{31}$

Si bien la hipotermia se ha propuesto como una técnica segura y eficaz, queda pendiente determinar si los pacientes que no cumplen criterios por no haberse incluido en los ensayos clínicos practicados hasta la fecha podrían beneficiarse de esta terapia: recién nacidos pretérmino, neonatos con colapso neonatal temprano o episodio sumamente mortal en el posparto, infartos o hemorragias cerebrales. Tampoco se conoce aún si los pacientes con asfixia perinatal, con un grado de encefalopatía leve, se beneficiarían; incluso en 25\% de ellos se encontrarán hallazgos patológicos en la resonancia magnética practicada en la segunda semana de vida y secuelas neurológicas a los 18 meses. ${ }^{32}$

La principal limitación de este estudio es su carácter retrospectivo. Su principal fortaleza es la amplia muestra y la capacidad de aportar información acerca de la evolución a mediano y largo plazo en función de los resultados del electroencefalograma. Hacen falta estudios clínicos que identifiquen nuevos signos de mal pronóstico durante la terapia con hipotermia. La pérdida en el seguimiento de seis pacientes también supone una limitación que debe tomarse en cuenta.

\section{CONCLUSIONES}

La terapia con hipotermia controlada parece favorable, según lo reportado hasta el momento en distintos metanálisis. Pese a las limitaciones de este estudio descriptivo queda claro que hubo mejoría en la evolución de los pacientes con encefalopatía hipóxico-isquémica moderada-severa a quienes se aplicó la terapia con hipotermia.

\section{REFERENCIAS}

1. Kurinczuk JJ, et al. Epidemiology of neonatal encephalopathy and hypoxic-ischaemic encephalopathy. Early Hum Dev 2010; 86: 329-38. https://doi.org/10.1016/j. earlhumdev.2010.05.010

2. Liu L, et al. Global, regional, and national causes of child mortality: an updated systematic analysis for 2010 with time trends since 2000. Lancet. 2012; 379 (9832): 2151-61. https://doi.org/10.1016/s0140-6736(12)60560-1

3. Jacobs SE, et al. Whole-body hypothermia for term and near-term newborns with hypoxic-ischemic encephalopathy: a randomized controlled trial. Arch Pediatr Adolesc Med. 2011; 165 (8): 692-700. https://doi.org/10.1001/ archpediatrics.2011.43

4. Simbruner $\mathrm{G}$, et al. Systemic hypothermia after neonatal encephalopathy: outcomes of neo. nEURO.network RCT. Pediatrics. 2010; 126 (4): e771-778. https://doi. org/10.1542/peds.2009-2441 
5. Azzopardi D, et al. Effects of hypothermia for perinatal asphyxia on childhood outcomes. N Engl J Med. 2014; 371 (2): 140-9. doi 10.1056/NEJMoa1315788

6. García-Álix, Quero J, editores. Evaluación neurológica del recién nacido. 1a ed. Madrid: Díaz de Santos, 2010; 975-1027.

7. Sarnat HB, Sarnat MS. Neonatal encephalopathy following fetal distress. A clinical and electroencephalographic study. Arch Neurol. 1976; 33 (10): 696-705. https://doi. org/10.1001/archneur.1976.00500100030012

8. Martinez-Biarge $M$, et al. Outcomes after central grey matter injury in term perinatal hypoxic-ischaemic encephalopathy. Early Hum Dev. 2010; 86 (11): 675-82. https://doi. org/10.1016/j.earlhumdev.2010.08.013

9. Jin $\mathrm{G}$, et al. Development of a novel neuroprotective strategy: combined treatment with hypothermia and valproic acid improves survival in hypoxic hippocampal cells. Surgery. 2014; 156 (2): 221-28. https://dx.doi. org/10.1016\%2Fj.surg.2014.03.038

10. Lingam I, Robertson NJ. Magnesium as a Neuroprotective Agent: A Review of Its Use in the Fetus, Term Infant with Neonatal Encephalopathy, and the Adult Stroke Patient. Dev Neurosci. 2018; 40 (1): 1-12. https://doi. org/10.1159/000484891

11. Maiwald CA, et al. Effect of allopurinol in addition to hypothermia treatment in neonates for hypoxic ischemic brain injury on neurocognitive outcome (ALBINO): study protocol of a blinded randomized placebo-controlled parallel group multicenter trial for superiority (phase III). BMC Pediatr. 2019; 19 (1): 210. https://doi.org/10.1186/ s12887-019-1566-8

12. Filippi L, et al. Safety and efficacy of topiramate in neonates with hypoxic ischemic encephalopathy treated with hypothermia (NeoNATI): a feasibility study. J Matern Fetal Neonatal Med. 2018; 31 (8): 973-80. https://doi.org/10.1 080/14767058.2017.1304536

13. Nuñez-Ramiro A, et al. Topiramate plus cooling for hypoxicischemic encephalopathy: A randomized, controlled, multicenter, double-blinded trial. Neonatology 2019; 116: 76-84. https://doi.org/10.1159/000499084

14. Juul SE, et al. High-Dose erythropoietin for asphyxia and encephalopathy (heal): A randomized controlled trial. Background, aims, and study protocol. Neonatology. 2018; 113 (4): 331-8. https://doi.org/10.1159/000486820

15. Moral Y, et al. Hipoxia-isquemia neonatal: bases celulares $y$ moleculares del daño cerebral y modulación terapéutica de la neurogénesis. Rev Neurol 2019; 68 (01): 23-36. https:// doi.org/10.33588/rn.6801.2018255

16. Graham EM, et al. Blood biomarkers for evaluation of perinatal encephalopathy: state of the art. Curr Opin Pediatr. 2018; 30 (2): 199-203. https://dx.doi.org/10.1097\%2FM OP.0000000000000591

17. Benavente-Fernandez I, et al. Altered plasma-type gelsolin and amyloid- $\beta$ in neonates with hypoxic-ischaemic encephalopathy under therapeutic hypothermia. J Cereb Blood Flow Metab. 2018; 39 (7): 1349-54. doi: 10.1177/0271678X18757419.

18. Basu SK, et al. Hypoglycaemia and hyperglycaemia are associated with unfavorable outcome in infants with hypoxic ischaemic encephalopathy: a post hoc analysis of the CoolCap Study. Arch Dis Child Fetal Neonatal Ed. 2016; 101 (2): F149-55. https://doi.org/10.1136/archdischild-2015-308733

19. Dageville $\mathrm{C}$, et al. Very early neonatal apparent life- threatening events and sudden unexpected deaths: incidence and risk factors. Acta Paediatr. 2008; 97 (7): 866-69. https:// doi.org/10.1111/j.1651-2227.2008.00863.x

20. Foran $A$, et al. Patterns of brain injury and outcome in term neonates presenting with postnatal collapse. Arch Dis Child Fetal Neonatal Ed. 2009; 94 (3): F168-77. https:// doi.org/10.1136/adc.2008.140301

21. Cornet $\mathrm{MC}$, et al. Use of therapeutic hypothermia in sudden unexpected postnatal collapse. Arch Pediatr. 2014; 21 (9): 1006-10. https://doi.org/10.1016/j.arcped.2014.06.009

22. Pejovic NJ, Herlenius E. Unexpected collapse of healthy newborn infants: risk factors, supervision and hypothermia treatment. Acta Paediatr. 2013; 102 (7): 680-88. https:// dx.doi.org/10.1111\%2Fapa.12244

23. Filippi $L$, et al. Incidence of sudden unexpected postnatal collapse in the therapeutic hypothermia era. Am J Perinatol. 2017; 34 (13): 1362-67. https://doi. org/10.1055/s-0037-1603678

24. Marin N, et al. Severe apparent life-threatening event during "skin-to-skin": treatment with hypothermia. An Pediatr (Barc). 2013; 79 (4): 253-56. https://doi.org/10.1016/j. anpedi.2013.06.009

25. Austin T, et al. To cool or not to cool? Hypothermia treatment outside trial criteria. Arch Dis Child Fetal Neonatal Ed. 2013; 98 (5): F451-53. https://doi.org/10.1136/ archdischild-2012-302069

26. Del Río R, et al. Amplitude integrated electroencephalogram as a prognostic tool in neonates with hypoxicischemic encephalopathy: A systematic review. PLoS One. 2016; 11 (11): e0165744. https://doi.org/10.1371/journal. pone. 0165744

27. Shankaran $S$, et al. Neonatal magnetic resonance imaging pattern of brain injury as a biomarker of childhood outcomes following a trial of hypothermia for neonatal hypoxic-ischemic encephalopathy. J Pediatr. 2015; 167 (5): 987-93.e983. https://doi.org/10.1016/j.jpeds.2015.08.013

28. Rollins N, et al. Predictive value of neonatal MRI showing no or minor degrees of brain injury after hypothermia. Pediatr Neurol. 2014; 50 (5): 447-51. https://doi.org/10.1016/j. pediatrneurol.2014.01.013

29. Cheong JL, et al. Prognostic utility of magnetic resonance imaging in neonatal hypoxic-ischemic encephalopathy: substudy of a randomized trial. Arch Pediatr Adolesc Med. 2012; 166 (7): 634-40. 
30. Liauw L, et al. Is there a way to predict outcome in (near) term neonates with hypoxic-ischemic encephalopathy based on MR imaging? AJNR Am J Neuroradiol. 2008; 29 (9): 1789-94. doi. 10.3174/ajnr.A1188

31. Weeks LC, et al. A novel magnetic resonance imaging score predicts neurodevelopmental outcome after perinatal asphyxia and therapeutic hypothermia. J Pediatr. 2018; 192: 33-40.e32.

32. Conway JM, et al. Mild hypoxic ischaemic encephalopathy and long-term neurodevelopmental outcome. A systematic review. Early Hum Dev. 2018; 120: 80-87. https://doi. org/10.1016/j.earlhumdev.2018.02.007 